\title{
Multilinguales
}

\section{Contact de langues et transferts conceptuels}

Contact of languages and conceptual transfers

\section{Marie Luce Honeste}

\section{(2) OpenEdition}

\section{Journals}

Édition électronique

URL : http://journals.openedition.org/multilinguales/1550

DOI : $10.4000 /$ multilinguales. 1550

ISSN : 2335-1853

\section{Éditeur}

Université Abderrahmane Mira - Bejaia

\section{Édition imprimée}

Date de publication : 1 juin 2014

Pagination : $5-20$

ISSN : 2335-1535

\section{Référence électronique}

Marie Luce Honeste, «Contact de langues et transferts conceptuels », Multilinguales [En ligne], 3 |

2014, mis en ligne le 03 juin 2014, consulté le 17 septembre 2019. URL : http://

journals.openedition.org/multilinguales/1550 ; DOI : 10.4000/multilinguales.1550

Ce document a été généré automatiquement le 17 septembre 2019.

\section{(c) (i) ()}

Multilinguales est mise à disposition selon les termes de la Licence Creative Commons Attribution Pas d'Utilisation Commerciale - Pas de Modification 4.0 International 


\title{
Contact de langues et transferts conceptuels
}

Contact of languages and conceptual transfers

\author{
Marie Luce Honeste
}

1 Comme le souligne l'appel à contribution pour ce numéro de la revue Multilinguales, « la langue, qui organise le réel, est un fait social inséparable de la culture ». On peut même considérer que la langue est le pivot de la culture, en ce qu'elle est, à la fois le reflet du cadre culturel dans lequel elle se développe et le moyen d'exprimer cette culture (Honeste, 2002).

2 Ce constat amène deux conséquences pour l'analyse des langues. La première est que le système grammatical et lexical d'une langue est un lieu privilégié d'observation des cadres culturels et conceptuels fondant l'identité d'une communauté de locuteurs. La seconde est que tout contact de cultures produit nécessairement un conflit de représentations culturelles générant à son tour des transformations systémiques dans les langues en contact.

Les langues concernées deviennent le lieu d'un mouvement réciproque d'assimilation des concepts culturels des communautés impliquées, entraînant un va-et-vient des signifiés et/ou des signifiants d'une langue à l'autre.

4 Cette étude propose une illustration de ces phénomènes de transferts culturels modifiant les langues en contact, à travers deux exemples: le premier pris dans le français du Liban, où se sont trouvés en contact l'arabe, langue de la culture musulmane historique, et le français, langue du protectorat français; le second pris dans le français de France qui, à l'instar de beaucoup d'autres langues aujourd'hui, se voit modifier de façon grandissante sous l'influence du modèle anglo-saxon. 


\section{Le cadre théorique : langue et rapport de l'homme au monde}

Dans une approche constructiviste de la linguistique et en particulier de la sémantique lexicale, dans la lignée des structuralistes comme Saussure et Guillaume (2002), on considère que le monde réel, extérieur à l'homme, est un " désordre " qui ne s'ordonne que sous le regard de l'homme qui en fait l'expérience (p.124.1-124.7). Chaque expérience partagée par une communauté humaine va se structurer collectivement dans un concept, qui va ensuite se stabiliser dans la langue sous la forme d'une unité lexicale. Il en résulte que le « signifié de langue » d'un mot n'est pas la description d'un phénomène du monde, mais le concept issu de l'expérience qu'en a fait la communauté. Ainsi chaque concept se forme dans le cadre culturel propre à une communauté de locuteurs, différent de tous les autres.

6 Cette approche conceptuelle du sens a une conséquence majeure en lexicologie, que nous avons développée ailleurs (Honeste, 2006) : le signifié de langue d'un mot n'est pas "polysémique » (ladite polysémie n'étant que la liste des descriptions des différents types de choses qu'il peut désigner), mais véhicule en langue un concept unique applicable à tous les types de choses susceptibles d'être appréhendées par ce même concept, quels que soient les domaines d'expérience dont elles relèvent.

7 Par ailleurs, dès lors qu'on admet que les mots véhiculent des concepts culturels et non des descriptions des choses du monde, on comprend pourquoi ces concepts, contrairement à des descriptions d'objets, varient selon les cultures qui les engendrent : en effet, si toutes les cultures ne faisaient que décrire le monde tel qu'il est, les mots signifieraient la même chose dans toutes les langues : seuls leurs signifiants seraient différents, tandis que leurs signifiés seraient identiques... et les traductions automatiques ne donneraient pas les résultats aberrants qu'on connaît.

Les transformations linguistiques observées en situation de contact de langues peuvent alors être analysées en termes de modifications des visions du monde, par assimilation de certaines conceptualisations de la culture de l'autre, comme l'illustrent les deux exemples développés à la suite.

\section{La dénotation de la vieillesse dans le français du Liban'}

9 On part de l'observation d'emplois de l'adjectif grand en français du Liban ne correspondant pas à ceux du français de France :

- en français du Liban :

Cet homme est grand. Mes parents sont grands. Mon mari est plus grand que moi.

- en français de France :

11 Cet homme est vieux. Mes parents sont vieux. Mon mari est plus âgé que moi.

12 Ces emplois spécifiques de grand sont des calques de l'arabe ${ }^{2}$. Ce qui nous intéresse ici, c'est de montrer que le remplacement de vieux par grand est la solution donnée par les Libanais francophones à un conflit de représentation de la notion de vieillesse dans les deux cultures en contact: conserver la représentation culturelle de la culture 
source, véhiculée dans le mot arabe ميبك [kabîr], dans un mot de la langue cible dont le concept soit le plus proche possible, dont le mot français grand est le véhicule.

La méthode suivie, que nous avons détaillée ailleurs (par exemple Honeste, 2006), consiste à établir le schéma conceptuel de chaque mot concerné, qui constitue son signifié unique de langue, à partir des constantes sémantiques et structurelles relevées dans tous ses emplois de discours. Pour chaque mot, à partir des différents sens recueillis dans les dictionnaires, des emplois en expression figée et des différents contextes possibles d'emploi, on repère, d'une part, les « domaines d'emploi » du mot (i. e. les différents domaines d'expérience, spatiaux, temporels ou notionnels, dans lesquels le mot est potentiellement utilisable); d'autre part, les "traits sémantiques constants ", présents dans tous les types d'emploi du mot (en évacuant les autres traits qui, selon notre approche théorique, appartiennent au contexte d'emploi et non au signifié propre du mot). À partir de ces deux relevés, comprenant des éléments cognitifs, culturels, sémantiques, grammaticaux, nous pouvons alors construire le schéma conceptuel du mot, qui constitue son signifié unique de langue.

14 Nous présentons ci-dessous un bref résumé ${ }^{3}$ des résultats de l'étude des trois adjectifs concernés, à savoir l'adjectif arabe بيبك [kabîr] et les deux adjectifs français grand et vieux.

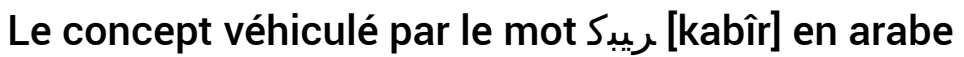

15 L'adjectif est présent dans les trois domaines d'emploi :

- le domaine spatial, où il s'applique à toute chose qui dépasse la norme ou la mesure ordinaire en raison de ses dimensions, sa quantité, son volume (notions de croissance, augmentation, grande taille, corpulence), ou son importance hiérarchique (partie principale d'un ensemble) ;

- le domaine temporel, où il s'applique à toute personne qui est d'un âge supérieur à une autre ;

- le domaine notionnel où il s'applique à tout ce qui est supérieur, important socialement ( éminence, grand personnage, grande entreprise); il caractérise des situations ou actions humaines dépassant la norme, difficiles, graves, intolérables (grand crime, péché) ; comme nom, il désigne la supériorité, l'importance morale ou sociale (grandeur, majesté, noblesse, prestige, fierté, orgueil) ou le sentiment d'importance (morgue, mépris).

Ses traits sémantiques constants sont la " position supérieure ».

Le concept qu'il véhicule peut être résumé comme suit :

- sa configuration cognitive est la localisation d'une position haute dans un espace gradué ou hiérarchisé ;

- son substrat culturel rend compte d'une représentation fondamentale de la culture arabe : l'association d'une symbolisation positive à la position supérieure dans un domaine hiérarchisé. Dans son application expérientielle à l'humain, le résultat est une forte charge positive sur la position du dominant, entraînant, chez le dominant, des sentiments d'orgueil, de fierté et de mépris vis-à-vis du dominé ; et, chez le dominé, la reconnaissance du statut de dominant et l'admiration de ce dernier. Le mot peut alors dénoter les plus grandes qualités morales et les plus hauts statuts sociaux: les personnages éminents en termes de pouvoir politique, social, intellectuel, aussi bien que les plus avancés en âge. 
18 L'insertion des hommes âgés dans ce statut supérieur est l'indice d'un trait culturel spécifique : le respect et la vénération apportés au grand âge en raison de sa tension vers la perfection, comme aboutissement de l'expérience. Il s'agit d'une notion essentiellement qualitative, évaluative à dominante positive.

\section{Le concept véhiculé par le mot grand en français de France}

Il est aussi présent dans les trois domaines d'emploi :

- dans le domaine spatial, il s'applique à toute chose qui dépasse la norme ou la mesure ordinaire en raison de ses dimensions, de sa quantité, de sa taille ;

- dans le domaine temporel, il dénote l'antériorité dans les liens de parenté (grand-père) ;

- dans le domaine notionnel, il dénote ce qui dépasse la norme dans de nombreux domaines : objets (grande route, grandes lignes, grande surface, grandiose) ; situations (grand silence, grande erreur, grand danger); statut social (grandeur, grand seigneur, grand personnage); comportement, soit en positif : qualités (grand homme, grand écrivain, grand couturier), soit en négatif : défauts (grand criminel), état psychologique (grande confusion, grand malade), domaine intellectuel (grands principes).

Ses traits sémantiques constants sont la " position supérieure ».

Le concept qu'il véhicule peut être résumé comme suit :

- sa configuration cognitive est la représentation d'une position haute dans un espace gradué ou hiérarchisé ;

- son substrat culturel inscrit la notion dans un système de représentation graduée qui structure de nombreux domaines d'expérience, d'où la grande fréquence d'emploi du mot. Une symbolique positive lui est associée dans les domaines où la gradation est hiérarchisée, comme le statut social ou les qualités morales. Mais les domaines d'évaluation quantitative sont plus nombreux que ceux d'évaluation qualitative.

On notera qu'il n'y a pas d'applications directes à l'âge : en effet, les seuls contextes d'emploi impliquant l'âge sont non évaluatifs, concernant une comparaison de taille liée à la croissance physique des enfants.

\section{Le concept véhiculé par le mot vieux en français de France}

Cet adjectif n'est présent que dans deux domaines d'emploi :

- le domaine temporel où il s'applique à ce qui est âgé (un vieil homme) [antonyme : jeune] ou ancien (vieille ville) [antonyme : nouveau]

- le domaine notionnel où il s'applique à ce qui est expérimenté (vieux routier); stable (vieil ami) ; dégradé physiquement, psychiquement, moralement (vieux fossile, vieux fou, vieux cochon), matériellement (vieille maison, vieux tacot, vieillerie).

Ses traits sémantiques constants sont l'« état d'ancienneté ». Le concept qu'il véhicule peut être résumé comme suit :

- sa configuration cognitive est la représentation d'un état localisé par rapport à un repère temporel ultérieur ;

- son substrat culturel construit une représentation plus symbolisée que celle de grand : l'état "vieux » peut être repéré, quantitativement, par rapport à "plus jeune ", mais aussi qualitativement; l'image culturelle est alors dissymétrique, avec un petit versant positif, 
avec prégnance de l'expérience et de la stabilité, et un grand versant négatif, indice d'une représentation culturelle plutôt négative de la vieillesse comme dégradation.

\section{Le processus de réajustement lexical en français du Liban}

Si on confronte les fonctionnements des trois adjectifs concernant la dénotation de l'âge, on constate alors que :

- du point de vue référentiel, ريبر [kabîr] dénote l'âge ; vieux est le mot le plus couramment employé pour dénoter l'âge ; grand ne dénote pas directement l'âge ;

- du point de vue conceptuel, vieux et ريبر [kabîr] présentent des conceptualisations de la vieillesse diamétralement opposées du point de vue de la symbolisation : vieux souligne les effets négatifs du temps (dégradation), ريبر[kabîr] ses effets positifs (expérience, sagesse, tension vers la perfection); en revanche, grand est le plus proche de ريبرك mots présentant la même perception positive du point de vue de la supériorité de la position.

On comprend alors que le mot grand ait été choisi en raison de sa proximité conceptuelle avec ريبك [kabîr], et non par rapport à son fonctionnement référentiel, puisqu'il n'est pas employé en français pour dénoter l'âge: en effet, si le critère référentiel avait présidé au choix, on aurait opté pour vieux ${ }^{4}$.

Du point de vue des conséquences du contact des langues, on constate que l'arrivée du français colonial dans un substrat culturel arabo-musulman a généré un conflit de représentations culturelles, en l'espèce à propos de la vieillesse : le mot français reflète une vision culturelle plutôt négative de la vieillesse comme dégradation, tandis que le mot arabe en donne une vision plutôt positive de supériorité vénérable. La résolution linguistique de ce conflit culturel a été une reconfiguration du micro-système de dénotation de la vieillesse dans le système lexical du français du Liban, où le mot grand prend les emplois de vieux ${ }^{5}$. On notera que la solution linguistique s'est faite au profit de la représentation culturelle locale, puisque c'est le concept arabe qu'on s'est efforcé de reproduire en cherchant un mot français qui en soit un équivalent conceptuel acceptable.

\section{Transfert lexical de problème à souci en français de france $^{6}$}

Depuis 2000, on assiste en français de France à un développement exponentiel des transferts d'emploi de problème à souci, souci prenant la place de problème dans la plupart des emplois du mot problème. Nous examinerons d'abord le phénomène du point de vue de ses réalisations en français et de ses effets sémantiques et conceptuels; puis nous nous intéresserons à l'origine du phénomène et à ses conséquences sur le plan culturel.

\section{Niveau intralinguistique : de problème à souci, un transfert conceptuel}

On entend couramment aujourd'hui employer souci à la place de problème, dans des expressions courantes comme " pas de souci! ", "Il y a un souci? / Quel est le souci ? J'ai un 
souci!». On pourrait imaginer que souci soit en passe d'évincer définitivement problème ; en réalité il n'en est rien, car, à y regarder de plus près, ce phénomène s'observe dans certains emplois seulement : l'examen qui suit permettra de mettre en évidence les raisons du phénomène.

Examinons en préalable le signifié de ces deux mots, établi à partir de leurs définitions lexicographiques. Une fois opéré le tri entre les éléments de sens provenant du concept et ceux provenant du contexte, on obtient les éléments sémantiques et morphosyntaxiques suivants, structurant le concept de langue (voir supra «le cadre théorique ») :

- problème (TLF) : « question à résoudre; difficulté $^{7}$ ".

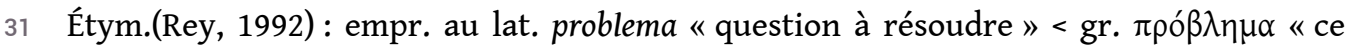

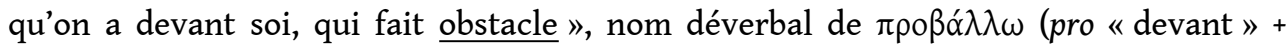
ballein « jeter ») « jeter devant ; mettre en avant comme argument ; proposer ».

On retient le substrat étymologique rémanent ${ }^{8}$ " obstacle", d'où découle la valeur conceptuelle « question à résoudre, difficulté ».

- souci (TLF) : « état d'esprit plus ou moins douloureux de quelqu'un qui s'inquiète à propos d'une personne ou d'une chose à laquelle il accorde de l'importance ».

Étym. (Rey, 1992) : nom déverbal de soucier < lat. vulg. *sollicitare « inquiéter, troubler, tourmenter ".

On retiendra de même pour souci le substrat étymologique "tourment ", d'où découle la valeur conceptuelle « état d'esprit d'inquiétude, de trouble ».

De ce bref résumé du sens lexical, on peut tirer une première conclusion : problème et souci ne sont pas "synonymes $»^{9}$; s'ils renvoient tous deux à une même notion de "difficulté ", problème dénote le phénomène qui est la cause de la difficulté, tandis que souci dénote l'état qui est la conséquence de la difficulté sur l'experiencer.

On constate par ailleurs que souci ne remplace pas toujours problème. Comprendre pourquoi permettra de mesurer les conflits conceptuels qui existent entre ces deux mots: pour cela, il faut examiner leurs emplois de discours afin de déterminer précisément où la substitution est possible et où elle ne l'est pas. Nous donnons ici nos résultats regroupés en fonction des divers critères de conflit d'emploi que nous avons pu dégager :

- du point de vue de la détermination, dans l'opposition massif / comptable :

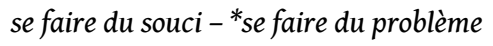

36 On observe l'emploi possible de souci avec le partitif, parce qu'il s'agit d'une notion susceptible d'être perçue comme massive, comme le sont en général les noms d'état et de sentiment; en revanche, cet emploi est impossible pour problème parce qu'il s'agit d'une notion perçue comme discrète (comptable), comme le sont en général les substances.

- du point de vue de la combinatoire, dans l'opposition objectif / subjectif :

poser des problèmes -*poser des soucis (vs causer des soucis)

On constate une compatibilité de problème, en position de complément d'objet, avec un verbe présentant l'objet comme externe au sujet (poser, présenter, etc.) : c'est l'indice d'une orientation externe, objective de la notion; en revanche, cet emploi est impossible pour souci, indice d'une orientation interne, subjective. Cette différence d'orientation est d'ailleurs implicitement prise en compte par les dictionnaires, qui 
glosent problème objectivement, comme "question à résoudre; difficulté ", et inversement souci subjectivement, comme « état d'esprit ».

Cette même perception objective de problème permet son emploi métonymique dans les domaines scientifique et technique, interdit à souci : problème d'astronomie, d'algèbre - *souci d'astronomie, d'algèbre

- du point de vue de la perception du référent, dans l'opposition abstrait / concret :

i. les problèmes techniques : « les difficultés d'ordre technique à résoudre lors de la réalisation de quelque chose». La représentation de la source de difficulté est abstraite (générique, globale), parce qu'elle est extérieure à l'expérience du sujet ;

ii. les soucis techniques: «les dysfonctionnements des machines». La représentation de la source de difficulté est concrète (spécifique, locale), parce qu'elle est intégrée à l'expérience du sujet.

- du point de vue de la syntaxe, dans l'opposition collectif / individuel :

i. expressions avec problème / souci en emploi absolu :

Il y a un problème / souci - Quel est le problème / souci? - Pas de problème / souci! Avoir un problème / souci - Avoir des problèmes / soucis

On constate que, lorsque la source de la difficulté n'est pas précisée, souci peut se substituer à problème dans tous les cas ;

ii. expressions avec problème / souci + complément introduit par de (structure [souci, problème de N]), où le complément dénote la source de difficulté :

( $\alpha$ ) le problème / ?le souci de l'éducation des enfants; avoir des problèmes / ?soucis d'argent, de santé;

( $\beta$ ) les problèmes / *soucis politiques, économiques, sociaux, etc.; le problème / * souci du désarmement, de la circulation urbaine, etc. ; les grands problèmes / ${ }^{*}$ soucis.

Lorsque la source de la difficulté est identifiée (par le complément), souci ne peut plus se substituer systématiquement à problème : en $(\alpha)$, où la source est de nature individuelle, la substitution est plus ou moins acceptable; en ( $\beta$ ), où la source est de nature collective, la substitution n'est pas possible. On déduit de ces observations qu'il existe des contraintes dues à la nature de la source : plus les difficultés sont d'ordre général ou collectif, moins la substitution par souci est acceptable. La raison en est que la perception collective efface la perception individuelle et la subjectivisation de la difficulté.

Le bilan qu'on peut faire de la confrontation des emplois des deux mots est conforme à celui issu des gloses lexicographiques : ces deux mots se situent dans un même champ notionnel, celui de l'expérience humaine de l'obstacle qui constitue une difficulté pour le sujet experiencer. Cependant, ils ne sont nullement synonymes, en ce qu'ils conceptualisent très différemment cette expérience commune :

- problème renvoie à un phénomène extérieur au sujet qui constitue une difficulté ( $c f$. étym. « obstacle ») : il est " orienté objet », dénotant la cause de la difficulté (le stimulus); il relève donc d'une approche objective de l'obstacle, qui autorise la combinatoire poser (des) problème(s);

- souci renvoie à un état "douloureux" du sujet (cf. étym. "tourment»), causé par un phénomène perturbant: il est "orienté sujet», dénotant l'effet de la difficulté sur l'experiencer (la réponse), et relève donc d'une approche subjective de la difficulté que produit l'obstacle, qui interdit la combinatoire * poser (des) souci(s). 
On comprend alors que les glissements d'emploi de problème à souci ne constituent pas une simple substitution de signifiants, qui serait due à un phénomène de "mode " ${ }^{10}$. Bien au contraire, ces glissements ne sont ni accidentels, ni anodins, car ces deux mots véhiculent des conceptualisations différentes de l'expérience humaine de la difficulté, et le remplacement de problème par souci est à comprendre au niveau intralinguistique comme un transfert conceptuel. En conformité avec les concepts que véhiculent ces mots, on peut avancer que ce glissement lexical est l'indice d'un recentrage de l'expérience de la difficulté sur le sujet, la difficulté étant non plus perçue comme « chose regardée » (Guillaume, 2002), mais seulement du point de vue de la sensation qu'elle suscite chez le « sujet regardant » (ibid.). Cette perception de l'effet et non de la cause est lourde d'implications :

- comme le montrent les gloses, un problème est « à résoudre » : il implique une attitude active du sujet consistant en une recherche de solution par investigation sur la cause, suivie autant que possible d'une action visant à l'éliminer ;

- un souci, en revanche, est un «état » du sujet: il n'implique en soi aucune recherche de solution et le sujet peut se contenter d'exprimer le sentiment en question. Néanmoins, il peut aussi chercher à éliminer ce sentiment, du fait qu'il est désagréable; l'action visera alors une opération pragmatique sur l'effet et non une investigation sur la cause.

\section{Niveau extralinguistique : de worry a souci, un transfert culturel}

Diverses études faites sur l'expression "pas de souci» attestent que les transferts observés en français ont une cause étrangère, qui est l'influence de l'anglais worry.

Nous procédons comme pour problème et souci, par l'examen préalable du signifié de worry à partir de ses définitions lexicographiques, dont nous conservons les éléments sémantiques et morphosyntaxiques suivants : « feel or cause to feel anxious or troubled about actual or potential problems" (Oxford Dictionary, s. v. "worry»). La définition indique que le mot dénote un sentiment de trouble ou d'anxiété et introduit la cause de ce sentiment par le mot problem : worry dénote donc un état qui est la conséquence d'une difficulté sur un experiencer, concept proche de celui de souci.

C'est précisément l'expression « No worries » qui est à l'origine de ces transferts : nous allons examiner rapidement le déroulement du processus. Selon Bruce Moore ${ }^{11}$, l'expression "no worries» est attestée en anglais australien depuis 1967. Son succès a été tel qu'elle a pris très vite des allures de devise nationale de l'Australie, arborée sur les plaques de voiture, à l'instar du " Je me souviens » québécois. Un tel succès s'explique par le fait que les Australiens y ont reconnu un trait prégnant de leur culture : Anna Wierzbicka classe l'expression parmi les australianismes les plus typiques et l'analyse comme un ethos majeur de la culture australienne ${ }^{12}$. L'expression gagne la GrandeBretagne au début des années 90, par le biais de séries télévisées australiennes, puis le Canada et les USA par la retransmission des Jeux Olympiques de Sydney (2000). En France, elle arrive dans les années 1990-2000 à travers son calque " pas de souci $»^{13}$. Cet anglicisme remplace très rapidement chez la quasi-totalité des locuteurs francophones l'expression "pas de problème», notamment par la vertu des nouveaux médias. Depuis 2000 , les emplois de souci ne cessent de s'étendre, souci se substituant à problème partout où c'est possible, compte tenu des contraintes conceptuelles soulevées supra.

Il est intéressant de souligner que la plupart des articles consacrés à l'étude de l'expression «pas de souci» l'interprètent systématiquement comme une formule 
incantatoire d'exorcisme de «La Crise». Cependant, son adoption rapide par la communauté francophone tout entière ${ }^{14}$ montre qu'elle relève de processus plus profonds touchant aux représentations mentales. Le fait que le glissement lexical de problème à souci s'observe, non point dans tous ses emplois, mais seulement là où une approche subjective de la difficulté est possible, est l'indice d'un transfert conceptuel. Mais il y a plus: le fait que ce phénomène, comme calque de "no worry", ait pour source une expression caractéristique de la culture anglo-saxonne est l'indice que derrière ce transfert conceptuel intralinguistique se cache un transfert culturel: l'expression apparaît comme une manifestation parmi beaucoup d'autres de l'envahissement grandissant, partout dans le monde, y compris dans la France cartésienne, d'une pensée anglo-saxonne, caractérisée par l'individualisme, la subjectivité (traits en conformité avec le concept que véhicule le mot souci, voir supra) et un optimisme requis en toutes circonstances. On est donc en droit d'y voir la manifestation linguistique d'une invasion plus large, celle d'un modèle de société (ultra)libérale, qui se veut global, fondé sur la seule relation marchande et qui a besoin, en conséquence, de tout centrer sur l'individu dans sa subjectivité, par une manipulation comportementale de type «positive attitude» comme logistique d'optimisation de la consommation.

On peut tirer de ces phénomènes linguistiques deux leçons.

48 La première concerne l'approche scientifique du fonctionnement sémantique des mots. Aussi bien dans le cas du transfert d'emploi de vieux à grand en français du Liban que de celui de problème à souci en français de France, on constate que c'est la configuration de la représentation mentale et non le fonctionnement référentiel qui a présidé à la modification lexicale du français. Cette observation non seulement valide et légitime une approche conceptuelle et non référentielle du signifié lexical, mais en outre, elle est l'indice que les locuteurs eux-mêmes, dans leur usage des mots, ont conscience à la fois du concept qu'ils véhiculent et de la prégnance de ce dernier sur le référentiel.

La seconde leçon est relative aux conflits de représentations culturelles générés par le contact de langues. Dans le cas du choix lexical de grand contre vieux, sous l'influence de l'arabe ميبـك[kabîr], nous avons vu que c'est le français de la colonisation qui assimile les traits culturels locaux prégnants au point de modifier son système conceptuel et donc ses emplois lexicaux. Le conflit de représentations est alors résolu au profit de la culture locale dont le vecteur est l'arabe. En revanche, dans le cas du choix lexical de souci contre problème sous l'influence de l'anglais worry, on assiste à un processus inverse : ce sont les cultures locales francophones qui empruntent à la culture anglo-saxonne son concept, de sorte que leurs emplois lexicaux s'en trouvent modifiés. Le conflit de représentations est alors résolu au profit de la culture anglosaxonne dont le vecteur est l'anglais. La différence entre les deux situations tient sans doute aux rapports de force entre les cultures en jeu: dans le cas du français de la colonisation dans le monde arabo-musulman, on constate que le substrat culturel local est resté suffisamment prégnant pour affecter la langue importée, au moins dans les lignes de force culturelles que constituent les structures sociales ${ }^{15}$.

50 À l'inverse, dans l'exemple du français de France, on voit que c'est le concept étranger qui s'impose, en l'occurrence, celui de l'anglais; la situation est différente, dans la mesure où l'anglais est ici, non plus la langue d'un groupe humain particulier, mais celle d'un modèle, celui du libéralisme économique, qui tend à imposer à l'ensemble de 
la planète un modèle unique de société de consommation, et ce à une vitesse à la mesure des nouveaux moyens de communication. Dans cette mesure, il affecte également toutes les langues et cultures de la planète.

\section{BIBLIOGRAPHIE}

ABDEL-NOUR, Al-Mufassal, Dictionnaire Arabe / Français, Beyrouth, 1983. AL-KAMEL, Al-Kanir, YOUSSEF, Reda, Dictionnaire de l'Arabe, Beyrouth, Librairie Liban Publishers, 1997.

BERNET, Charles, REZEAU, Pierre, On va le dire comme ça. Dictionnaire des expressions quotidiennes, Paris, Balland, 2008.

GIORGIONE, «'Y a pas de souci !', une expression disséquée », blog 24 heures Philo du 7 mars 2009, disponible sur : [http://philosophie.blogs.liberation.fr/about.html]

GRAND ROBERT ELECTRONIQUE, Paris, Le Robert, 1999, disponible sur Cédérom.

GUILLAUME, Gustave, «Textes inédits », in Le système des parties du discours. Sémantique et syntaxe. Actes du IXe colloque de l'Association internationale de psychomécanique du langage, Québec, Presses de l'Université Laval, 2002.

HONESTE, Marie Luce, « Organisation du lexique et identité culturelle », in B. LewandowskaTomaszczyk \& M. Thelen (éds), Translation and MeaningPart 6. Proceeding of the 3rd International Maastricht-Lodz Duo Colloquium, Lodz, Poland, 22-24/09/2000, Universitaire Pers Maastricht, The Netherlands, 2002, pp. 211-221.

HONESTE, Marie Luce, « Approche cognitive du sens lexical », in Actes du XXIVe Congrès International de Linguistique et de Philologie Romanes, Aberystwyth, 1er - 6 Août 2004, vol. IV, TROTTER David A. (dir.), Tübingen, Niemeyer Verlag, 2006, pp. 105-118.

HONESTE, Marie Luce, « Le français parlé libanais : une identité multi-culturelle », in DORLIAN G. (dir.), La Francophonie : conflit ou complémentarité identitaire ?, vol. 1, Tripoli (Liban), Publications de l'Université de Balamand, 2008, pp. 451-465.

HONESTE, Marie Luce, « Le phénomène de rémanence et ses conséquences en sémantique lexicale à travers l'histoire du mot opinion ", in Le Français Préclassique n 13, Champion, 2011, p. 91-113.

HONESTE, Marie Luce, « Sémantique conceptuelle et synonymie », in La Synonymie, Université Paris IV-Sorbonne, Françoise Berlan \& Gérard Berthomieu (dir.), Paris, PUPS, 2012, pp. 59-71.

HONESTE, Marie Luce, «Les transferts de représentations dans les langues : de worry à souci et de problème à souci, un exemple de calque culturel en français de France ", in KACPRZAK Alicja, KONOWSKA Agnieszka, GAJOS Mieczysław (dir.), Pluralité des cultures : chances ou menaces. Analyses linguistiques et didactiques, Oficyna Wydawnicza LEKSEM, Łódź - Łask 2012, Publikacja dofinansowana przez UrzĄd Miasta Łódź, 2012, pp. 155-164.

OXFORD DICTIONARIES ONLINE, disponible sur [http://oxforddictionaries.com REIG, Daniel, Dictionnaire Arabe-Français, Français-Arabe, Paris, Larousse, 1999.

ROBIN, Jean-Pierre, « Pas de souci, le remède des français face à la crise », in Le Figaro, 11 décembre 2009. 
SAUSSURE, Ferdinand de, Cours de Linguistique Générale, Paris, Minuit, [1916] 1968.

SAYAH, Antoine, « Expressions françaises d'origine libanaise dans L'Orient-Le Jour », in Bilinguisme, traduction et Francophonie, 9ème Sommet de la Francophonie, Beyrouth, 2001, Kaslik (Liban), 2002,

pp. 227-231.

TRESOR DE LA LANGUE FRANÇAISE. P. Imbs \& B. Quemada (dir.),

Dictionnaire de la langue du xixe et du xixe siècle (1789-1960), Nancy, CNRS-INALF, \& Paris, Gallimard, 16 vol. , 1971-1994.

TLFi, disponible sur [http://atilf.atilf.fr/tlf.htm].

WIERZBICKA, Anna, Semantics, Culture, and Cognition : Universal Human Concepts, in Culture-specific Configurations, New York, Oxford University Press US, 1992.

\section{NOTES}

1. Les résultats complets de cette étude ont été présentés en 2008 dans un article intitulé «Le français parlé libanais: une identité multi-culturelle», in La Francophonie: conflit ou complémentarité identitaire?, G. Dorlian (éd.), Publications de l'Université de Balamand, Tripoli (Liban), vol. 1, p. 451-465.

2. Merci à MM. Nader Srage, Docteur en Sociolinguistique à l'Université Libanaise, et Hassam Hamzé, Professeur de Linguistique Arabe à l'Université Lumière Lyon 2 (France), pour leur aide précieuse concernant l'arabe et le français libanais.

3. Pour alléger la lecture, l'analyse complète n'est pas produite.

4. Le même processus explique que, pour dénoter la haute taille, le français du Liban a préféré utiliser l'adjectif long (plutôt que grand en français de France), dont le schéma conceptuel se rapproche plus de l'arabe لل يوط [tawîl] (voir Honeste, 2008).

5. Notons que le mot vieux ne disparaît pas en français du Liban, mais se trouve affecté aux mêmes emplois que l'arabe مريدق [qadìm], dont le schéma conceptuel se rapproche plus.

6. La totalité de cette étude a été publiée en 2012 sous le titre «Les transferts de représentations dans les langues: de worry à souci et de problème à souci, un exemple de calque culturel en français de France " (voir Références en fin d'étude).

7. Sont soulignés les éléments qui sont retenus pour construire le concept.

8. Sur le phénomène de rémanence, voir Honeste, 2011.

9. Sur la critique de la notion de synonymie, voir Honeste, 2012.

10. Contrairement à ce que suggère l'académicien Marc Fumaroli, Président de la commission de terminologie au sein de la Délégation Générale à la Langue Française, qui a commandé en 2009 à ses collaborateurs une enquête sur l'expression « pas de souci » dans cette perspective.

11. Chercheur au National Dictionary Centre de Camberra (Australie).

12. Ethos ainsi caractérisé : «[...] amiability, friendliness, an expectation of shared attitudes (a proneness to easy "mateship"), jocular toughness, good humour, and, above all, casual optimism »(Wierzbicka, 1992 : 388).

13. Aussi attesté dans une curieuse version hybride «no soucy» (prononcer [susaj]), lancée par la chanteuse française Ophélie Winter.

14. On constate qu'elle gagne rapidement toute la francophonie : je témoigne personnellement de son usage en Pologne et en Algérie; selon d'autres témoins, elle circule aussi chez les locuteurs francophones hors francophonie, comme en Espagne.

15. En revanche, dans les aspects superstructurels comme l'économie, on constate une invasion croissante, dans les langues locales, de concepts venus des modèles économiques européens, 
indice de l'assimilation desdits modèles (voir à ce sujet S. Lanseur, Le changement lexicosémantique dans le discours de l'économie à travers l'émission radiophonique Le rendez-vous de l'économie et le journal El Watan, Thèse de Doctorat, Université de Bejaia, Algérie, 2011).

\section{RÉSUMÉS}

Ce papier vise à illustrer les relations entre langue et culture à travers deux exemples. Le premier concerne le transfert d'emploi de vieux à grand en français du Liban, par l'intermédiaire de l'arabe ريبرك [kabîr]; le second concerne le récent transfert d'emploi de problème à souci en français de France. L'étude s'inscrit dans une théorie conceptuelle et cognitive du sens lexical dans laquelle les transferts apparaissent comme le résultat d'une modification des représentations culturelles en situation de contact de langues. Les phénomènes sont mis en relation avec leur cadre politique: pour le premier, le post-colonialisme, pour le second, la mondialisation du libéralisme.

This paper aims to illustrate the relations between culture and language, through two examples. The first one concerns the transfers of use from the word vieux to the word grand in French of Lebanon, through the medium of the Arabic word ريب [kabîr] ; the second one concerns the recent transfers of use from the French word problème to the word souci, through the medium of the English word worry.The study is based on a conceptual and cognitive theory of the lexical meaning, where transfers appear as the result of an alteration of the cultural representations in the case of code switching. Phenomena are connected to their political frame, i.e. for the first one, colonialism, and for the second one, the globalisation of liberalism.

\section{INDEX}

Keywords : lexicon, cognition, culture, code switching, conceptual transfers

Mots-clés : lexique, cognition, culture, contact de langues, transferts conceptuels

\section{AUTEUR}

\section{MARIE LUCE HONESTE}

Université de Rennes 2 Haute-Bretagne

Laboratoire : E.A. 4089 " Sens, Texte, Histoire ", Paris IV Sorbonne

Marie Luce SELOSSE-HONESTE est professeur des universités, émérite, en linguistique et littérature, à Université Rennes 2 Haute-Bretagne - UFR Arts, Lettres, Communication (ALC).

- Laboratoires de rattachement :

Sens, Texte, Informatique, Histoire ", École doctorale V, "Concepts et Langage", E.A. 4509, dir. Pr Joëlle Ducos, Université Paris IV Sorbonne, PREFics (Plurilinguismes, Représentations, Expressions Francophones - Information, Communication, Sociolinguistique), E.A. 3207, UMR LCF 8143, dir. Pr Catherine Loneux, Université Rennes 2 Haute-Bretagne, CRTT (Centre de Recherches en Terminologie et Traduction), E.A. 4162, dir. Pr François Maniez, Université Lyon 2 (Lumière). 
- Directions d'ouvrages collectifs :

Animots, Marie Luce Honeste \& Roger Sauter (éds), Saint-Etienne, Travaux LXXXVIII du CIEREC, Publications de l'Université de Saint-Étienne, 1996.

Dire l'Espace familier, Marie Luce Honeste (éd.), Saint-Etienne, Travaux C du CIEREC, Publications de l'Université de Saint-Étienne, 2000.

- Collaborations à des ouvrages,

Dictionnaire du français usuel : des 500 mots de base aux 10000 mots utiles, Jacqueline Picoche \& JeanClaude Rolland, éd. De Boeck \& Larcier S.A., département Duculot, 2001.

- Publications : une soixantaine de publications dont :

"Sémantique conceptuelle et synonymie ", in "La Synonymie », Université Paris IV-Sorbonne, dir. Françoise Berlan \& Gérard Berthomieu, PUPS, 2012, p. 59-71.

« Les transferts de représentations dans les langues : de worry à souci et de problème à souci, un exemple de calque culturel en français de France », in Pluralité des cultures : chances ou menaces. Analyses linguistiques et didactiques, Alicja Kacprzak, Agnieszka Konowska, Mieczysław Gajos (eds), Oficyna Wydawnicza LEKSEM, Łódź - Łask 2012, Publikacja dofinansowana przez UrzĄd Miasta Łódź, 2012, p. 155-164.

« Langue et contexte : deux sources de signification. L'exemple du verbe modal pouvoir », Editions Universitaires Européennes, Südwestdeutscher Verlag für Hochschulschriften, Sarrebruck (Allemagne), 2012, rééd. 2004.

« La Liste dans Le Quart Livre de Rabelais : (dés)organisation et (en)jeux discursifs », Le Français Préclassique $\mathrm{n}^{\circ}$ 15, Champion, 2012, rééd. 2004, p. 171-205.

« Ordre \& Désordre dans Delie De Maurice Scève (1544) », Le Français Préclassique n 15, Champion, nov. 2013, p. 207-222.

\section{En cours de parution}

«Les mots, les choses, les concepts : y a-t-il une différence entre nomenclature scientifique et lexique courant?", in Les mots et les choses au XVIIIe siècle : la science, 'langue bien faite'?, D. Reynaud \& Ph. Selosse (eds), 2014.

"Contact de langues et transferts conceptuels », Multilinguales $\mathrm{n}^{\circ} 3$, Les langues et cultures étrangères dans les discours interactifs oraux et écrits, Université Abderrahmane Mira Faculté des lettres et des langues, Laboratoire LAILEMM, 2014, Béjaia (Algérie). 